\title{
DÉTERMINATION \\ DE LA TENEUR EN SOLIDES NON-GRAS DU LAIT AVEC LES BILLES HYDROMĖTRES DE GOLDING (1)
}

\author{
par \\ Roger-N. GIROUX (2)
}

\section{Introduction}

La littérature technique décrit une méthode simple et rapide pour déterminer la teneur en solides non-gras dans le lait. Il s'agit de la méthode de Golding utilisant des billes hydromètres de plastique $[5,6,7,10]$. On a aussi tenté d'appliquer cette méthode à l'analyse des échantillons composés de quinzaine [2, 9].

On considère qu'il y aurait quatre principaux avantages à l'emploi d'une telle méthode.

1. - Une sélection au sein des races laitières, le niveau de la teneur en S.N.G. du lait relevant d'un facteur héréditaire distinct de celui pour la teneur en gras.

2. - L'Etablissement du prix du lait en fonction de sa composition globale.

3. - La détermination rapide des solides dans certains produits laitiers manufacturés.

4. - Un essai présomptif pour le décalage des laits mouillés, dont confirmation se ferait au cryoscope.

\section{Matériel}

L'équipement consiste en une série de bouteilles à échantillon en verre de 4 1/2 onces munies d'un couvercle métallique vissé. Dix billes de plastique de $5 / 16$ pouce de diamètre, de densité connue, voisine de la densité où la gravité spécifique du lait et de couleurs différentes sont placées dans les bouteilles.

Chacune des dix billes de plastique dans les bouteilles de verre est d'une densité normalisée à 0,001 unité de gravité spécifique près, de façon que sa descente soit juste initiée dans un lait ayant sa densité respective.

(1) Le Québec Laitier et Alimentaire, juillet 1964.

(2) Directeur technique adjoint, chef du Contrôle et des Recherches à la Coopérative agricole de Granby. 
TABLEAU 1

SPÉCIFICATIONS DES BILLES QUANT A LEUR COULEUR ET LEUR DENSTTÉ $\left(\mathrm{g} / \mathrm{ml}\right.$ à $\left.68^{\circ} \mathrm{F}.\right)[10]$

\begin{tabular}{lrrr}
\multicolumn{2}{c}{ Couleur } & & Densité \\
Vert pâle & 10 & 1.025 \\
Violet & 9 & 1.026 \\
Bleu & 8 & 1.027 \\
Rose & 7 & 1.028 \\
Aqua & 6 & 1.029
\end{tabular}

\begin{tabular}{ll}
\multicolumn{2}{c}{ Couleur } \\
Vert foncé & $\mathbf{5}$ \\
Violet & $\mathbf{4}$ \\
Bleu & $\mathbf{3}$ \\
Rose & $\mathbf{2}$ \\
Aqua & $\mathbf{1}$
\end{tabular}

Densité

Dès lors, chaque bille hydromètre d'une couleur particulière couvre une variation de densité de $0,001 \mathrm{~g} / \mathrm{ml}$ à $68^{\circ} \mathrm{F}$. S'il s'agit de la bille vert foncé ayant la densité de 1,030 , sa descente sera initiée dans un lait qui aura une densité de $1,030 \mathrm{~g} / \mathrm{ml}$ à $68^{\circ} \mathrm{F}$.

Evidemment, les lacto-densimètres, tel le Quévenne, sont calibrés pour faire la lecture de la gravité spécifique à $60^{\circ} \mathrm{F}$. Pour fin de comparaison, il faudrait faire la correction qui s'impose tenant compte de la différence entre densité et gravité spécifique, ainsi que tenant compte du facteur de correction pour la différence de température.

La méthode utilisant les billes serait avantageuse quant à la précision obtenue, comparativement au lactodensimètre de verre, parce que l'expansion thermale du plastique utilisé, contrairement à celle du verre, est à peu près la même que celle du lait entre 66 et $72^{\circ} \mathrm{F}$.

Pour éviter de perdre les billes en rejetant le lait, un treillis de plastique est posé à pression sur la large embouchure de la bouteille. On peut descendre une pipette à travers le treillis de plastique pour prélever tout échantillon devant servir à un autre essai, tel l'essai Babcock pour la teneur du gras.

\section{Méthode}

Un échantillon représentatif d'environ $50 \mathrm{ml}$ de lait est versé dans la bouteille munie du treillis et contenant déjà les dix billes, le contenu est chauffé à $104^{\circ} \mathrm{F}$. et maintenu à cette température 5 minutes, tout particulièrement s'il avait été refroidi antérieurement, et le contenu est refroidi à $68^{\circ} \mathrm{F}$. pendant au moins $2 \mathrm{mi}$ nutes. Bien agiter l'échantillon par un mouvement rotatif, ne pas agiter vigoureusement afin d'éviter le barattage. Le nombre de billes reposant au fond du contenant à une température donnée détermine la densité du lait. On peut utiliser un miroir posé à angle de 45 degrés, sousjacent à un support où sont placées les bouteilles permettant de voir facilement le fond des bouteilles. On peut vérifier en comptant le nombre de billes flottant à la surface. 
Les billes comptées au fond et en surface devraient former un total de 10, quoique occasionnellement une bille étant de la même densité que le lait, restera à mi-chemin entre le fond et la surface. Un nouveau mouvement rotatif pourra faire descendre au fond ou monter en surface la bille. Si le mouvement rotatif n'a pas été bien fait et si la majorité des billes descend au fond, il se peut qu'une bille s'arrête sur une autre, et on ne la verra pas bien au fond $\mathrm{du}$ contenant.

La quantité de lait placée dans la bouteille n'a pas à être mesurée. On peut placer jusqu'à $80 \mathrm{ml}$ de lait, admettant qu'on désirerait effectuer d'autres expertises sur l'échantillon de lait. Toutefois, on recommande que la hauteur du niveau de lait nesoit pas inférieure à un pouce.

On peut conserver les échantillons jusqu'à deux jours à 65$75^{\circ} \mathrm{F}$ si on prend le soin d'ajouter une goutte par $25 \mathrm{ml}$ de lait d'un préservatif de 0,25 p. 100 de résazurine comme colorant daris de la formaline, sans que la lecture des résultats ne soit influencée. La résazurine est réduite à la couleur rose dans la formaline. Si le lait s'était détérioré au point de sûrir, et que le mélange préservatif et lait avait définitivement perdu sa coloration rose, il faudrait conclure que les résultats ne sont pas utilisables.

L'intensité de la coloration rose indiquerait approximativement la quantité de formaline ajoutée et en préviendrait une addition excessive, laquelle peut rendre l'effectuation de l'essai Babcock plus difficile et faire perdre de la précision à la détermination de la densité, d'où la teneur en S.N.G.

Si on devait effectuer une détermination de protéine par absorption de colorant, puisque la formaline cause interférence dans cet essai, il ne faudrait pas l'employer.

Nous croyons que l'emploi des petits comprimés de préservatif que nous avons décrit précédemment [4] pourrait présenter certains avantages sur la formaline additionnée de résazurine quant à la préservation des échantillons.

\section{Lavage des contenants et billes hydromètres}

La façon de laver l'équipement est la suivante :

Enlever le couvercle métallique, et verser le lait tout en maintenant le treillis de plastique en place pendant tout le cours du lavage.

Rincer à l'eau froide. Laver dans de l'eau à environ $100^{\circ} \mathrm{F}$ utilisant un détergent rendu légèrement alkalin aveo du Calgon. Au besoin, laisser tremper 1 heure. Ne pas utiliser de savon.

Rincer deux fois à l'eau froide, puis une fois avec de l'eau distillée contenant $2-4 \mathrm{ml}$ de formaline par gallon. Renverser les bouteilles pour drainer et sécher sur un support qui permet une bonne circulation d'air. 
Pour usages successifs des bouteilles, bien rincer la bouteille avec l'échantillon de lait suivant.

\section{Expérimentation}

Nous nous sommes donc procuré un service de 12 bouteilles avec billes hydromètres de Golding en nous adressant à Udy Analyser Co., P.O. Box 148, Boulder, Colorado, U.S.A. Coût approximatif : $\$ 13,00$.

Des échantillons de lait prélevés dans le bassin de pesée à la réception de l'une de nos usines furent donc soumis à cette détermination de la teneur en solides non gras, en comptant le nombre de billes descendues au fond des bouteilles, et en faisant les déterminations de la teneur en gras de Babcock et des S.T. par la méthode Mojonnier, obtenant la teneur gravimétrique en S.N.G. par différence. La façon de convertir les résultats, en terme de nombre de billes au fond de la bouteille, à teneur en S.N.G. est la suivante.

Plusieurs formules ont été proposées pour déterminer la teneur en S.N.G. en partant de 1) la densité exprimée en $\mathrm{g} / \mathrm{ml}$ à $68^{\circ} \mathrm{F}$, ou la gravité spécifique à $60^{\circ} \mathrm{F}$ et 2) la teneur en gras obtenue par l'essai Babcock.

Dans un travail préliminaire, des chercheurs de l'Université de l'Etat de Washington (U.S.A.), ont compilé une formule à régression multiple basée sur 1873 échantillons de lait provenant de 53 troupeaux des races Guernesey, Molstein et Jersey, partant de la teneur en solides totaux par gravimétrie et la teneur en gras par l'essai Babcock sur des échantillons distincts de lait de vaches individuelles. Cette formule qui suit, présentée par Erb, Manus et Ashworth [3] est utilisée couramment par l'Association américaine d'amélioration des troupeaux laitiers (Dairy Herd Improvement Association).

S.N.G. $=9,13-(0,279 \times$ nbre de billes au fond $)(0,307 \times \% G)$

Un tableau fourni avec l'équipement ainsi que les instructions sur la façon de s'en servir, présente les valeurs précalculées par cette formule en fonction des variations de 0,1 p. 100 de la teneur en gras entre 2,0 et 9,9 p. 100 de gras et des variations de 0,03 p. 100 de la teneur en S.N.G. entre 6,96 et 12,17 p. 100 de S.N.G.

Nous croyons que deux restrictions s'imposent quant à l'emploi de ce tableau de conversion. Quoique le tableau présente directement les chiffres précalculés pour la teneur en solides non gras avec une précision au centième, puisque l'une des données sur laquelle les calculs sont basés n'a qu'une précision au dixième, soit la teneur en gras déterminée au Babcock, une bonne compréhension de la signification des nombres statistiques nous fait rejeter 
cette précision au centième, telle que présentée, pour ne retenir que la précision au dixième.

Par ailleurs, assurer que la teneur en gras de laits normaux pourrait varier de 2,0 à 9,9 p. 100 nous semble exagéré. Les extrêmes correspondraient à du lait de début et de fin de la traite, que nous ne pouvons considérer comme lait normal.

Nous recommandons done la restriction de l'emploi des chiffres présentés au tableau de conversion correspondant aux teneurs en gras variant de 2,5 à 6,5 p. 100 de gras plutôt que de 2,0 à 9,9 p. 100 de gras.

Les résultats d'expérience que nous avons obtenus sont présentés avec la précision au dixième seulement et non au centième, afin de tenir compte des restrictions ci-dessus proposées.

\section{Résultats}

Cinq séries de 12 observations obtenues pour un total de 60 échantillons furent recueillies les 9 (tableau 1), 10 et 11 (tableau 2), 12 (tableau 3) septembre, et 6 (tableau 4) novembre 1963.

Tous les échantillons furent en provenance de patrons différents excepté ceux où les observations furent répétées pour les 10 et

TABLEAU 2

RÉSULTATS DE DÉTERMINATION DE LA TENEUR EN S.N.G. PAR LA MÉTHODE GOLDING EN COMPARAISON A LA MÉTHODE GRAVIMETRIQUE (9 septembre 1963)

\begin{tabular}{|c|c|c|c|c|c|c|}
\hline \multirow[b]{2}{*}{ Numéro } & \multicolumn{3}{|c|}{$P A R \quad D I F F E R E N C E$} & \multicolumn{2}{|c|}{$G O L D I N G$} & \multirow[b]{2}{*}{ Différence } \\
\hline & $\begin{array}{l}\text { Gras \% } \\
\text { Babcock }\end{array}$ & $\begin{array}{c}\text { S.T. \% } \\
\text { (Mojonnier) }\end{array}$ & $\begin{array}{l}\text { S.N.G. \% } \\
(\text { Diffé- } \\
\text { rence) }\end{array}$ & $\begin{array}{l}\text { No de } \\
\text { billes } \\
\text { au fond }\end{array}$ & $\begin{array}{c}\text { S.N.G. \% } \\
\text { Corres- } \\
\text { pondants }\end{array}$ & \\
\hline 1 & 3,5 & 12,48 & 9,0 & 6 & 8,5 & 0,5 \\
\hline 2 & 3,2 & 12,05 & 8,8 & 5 & 8,7 & 0,1 \\
\hline 3 & 3,5 & 12,46 & 9,0 & 5 & 8,8 & 0,2 \\
\hline 4 & 3,0 & 11,62 & 8,6 & 6 & 8,4 & 0,2 \\
\hline 5 & 3,2 & 11,45 & 8,2 & 7 & 8,2 & 0,0 \\
\hline 6 & 3,1 & 11,82 & 8,7 & 6 & 8,4 & 0,3 \\
\hline 7 & 3,2 & 11,93 & 8,7 & 5 & 8,7 & 0,0 \\
\hline 8 & 3,4 & 12,14 & 8,2 & 6 & 8,5 & 0,2 \\
\hline 9 & 3,3 & 11,84 & 8,5 & 7 & 8,2 & 0,3 \\
\hline 10 & 3,5 & 12,56 & 9,1 & 5 & 8,8 & 0,3 \\
\hline 11 & 4,2 & 13,23 & 9,0 & 5 & 9,0 & 0,0 \\
\hline 12 & 4,1 & 13,10 & 9,0 & 5 & 9,0 & 0,0 \\
\hline
\end{tabular}


TABLEAU 3

RÉSUltats DE DÉtermination DE LA TENEUR EN S.N.G. PAR LA MÉTHODE GOLDING EN COMPARAISON A.LA MÉTHODE GRAVIMÊTRIQUE (10 et 11 septembre 1963)

\begin{tabular}{|c|c|c|c|c|c|c|}
\hline \multirow{2}{*}{ Numéro } & \multicolumn{3}{|c|}{ PAR DIFFÉRENCE } & \multicolumn{2}{|c|}{$G O L D I N G$} & \multirow{2}{*}{ Différence } \\
\hline & $\begin{array}{l}\text { Gras \% } \\
\text { Babcock }\end{array}$ & $\begin{array}{c}\text { S.T. \% } \\
\text { (Mojonnier) }\end{array}$ & $\begin{array}{l}\text { S.N.G. \% } \\
(\text { Diffé- } \\
\text { rence) }\end{array}$ & $\begin{array}{l}N^{\circ} \text { de } \\
\text { billes } \\
\text { au fond }\end{array}$ & $\begin{array}{c}\text { S.N.G. \% } \\
\text { Corres- } \\
\text { pondants }\end{array}$ & \\
\hline 1 & 4,4 & 13,72 & 9,3 & 6 & 8,8 & 0,5 \\
\hline 1 & 4,0 (1) & 13,29 & 9,3 & 5 & 8,7 & 0,6 \\
\hline 2 & 3,2 & 11,79 & 8,6 & 7 & 8,2 & 0,4 \\
\hline 2 & 3,2 (1) & 11,80 & 8,6 & 7 & 8,2 & 0,4 \\
\hline 3 . & 3,5 & 12,27 & 8,8 & 7 & 8,2 & 0,6 \\
\hline 3 & $3,4(1)$ & 12,12 & 8,7 & 6 & 8,5 & 0,2 \\
\hline 4 & 3,1 & 11,83 & 8,7 & 7 & 8,1 & 0,6 \\
\hline 4 & $3,2(1)$ & 12,22 & 9,0 & 6 & 8,4 & 0,6 \\
\hline 5 & 3,4 & 12,33 & 8,9 & 6 & 8,5 & 0,4 \\
\hline 5 & $3,3(1)$ & 12,39 & 9,1 & 6 & 8,5 & 0,6 \\
\hline 6 & 2,9 & 9,67 & 6,8 & 10 & $<7,2$ & $?$ \\
\hline 6 & $2,8(1)$ & 9,66 & 6,9 & 10 & $<7,2$ & $?$ \\
\hline 7 & 3,2 & 11,82 & 8,6 & 7 & 8,2 & 0,4 \\
\hline 7 & 3,6 (1) & 12,44 & 8,8 & 7 & 8,3 & 0,5 \\
\hline 8 & 3,8 & 12,91 & 9,1 & 6 & 8,6 & 0,5 \\
\hline 8 & 3,7 (1) & 12,79 & 9,1 & 6 & 8,6 & 0,5 \\
\hline 9 & 3,5 & 12,38 & 8,9 & 6 & 8,5 & 0,4 \\
\hline 9 & 3,3 (1) & 12,25 & 8,9 & 6 & 8,5 & 0,4 \\
\hline 10 & 3,4 & 12,33 & 8,9 & 6 & 8,5 & 0,4 \\
\hline 10 & 3,8 (1) & 12,86 & 9,1 & 6 & 8,6 & 0,5 \\
\hline 11 & 3,9 & 12,76 & 8,9 & 7 & 8,4 & 0,5 \\
\hline 11 & 3,9 (1) & 13,02 & 9,1 & 6 & 8,7 & 0,4 \\
\hline 12 & 3,3 & 12,35 & 9,0 & 6 & 8,5 & 0,5 \\
\hline 12 & 3,3 (1) & 12,28 & 9,0 & 6 & 8,5 & 0,5 \\
\hline
\end{tabular}

(1) Résultats jumelés obtenus à une journée d'intervalle pour le lait des mêmes patrons.

11 novembre, puisque les résultats furent obtenus pour du lait en provenance des mêmes patrons, mais à une journée d'intervalle. Ces résultats jumelés sont assez semblables. Dans tous ces résultats, la teneur en gras varia entre 3,0 et 4,2 , celle en S.N.G. gravimétrique entre 8,2 et 9,2 , et le nombre de billes au fond de la bouteille de 7 à 4 , ce qui correspond à des deńsités de 1,028 et 1,031 à $68^{\circ} \mathrm{F}$ respectivement.

Un fait intéressant à noter, c'est que le lait d'un patron, échantillon $n^{\circ} 6$, tableau 2, eut un comportement anormal. Basse teneur en gras, $2,8-2,9$, en solides totaux $9,66-9,67$, et en solides non gras 
6,8-6,9. Toutes les billes reposaient au fond de la bouteille, indiquant une densité inférieure à 1,025 à $68^{\circ} \mathrm{F}$.

Les 18 et 19 septembre, des échantillons de lait prélevés de ce patron, et soumis à la détermination du point de congélation par cryoscopie, indiquaient un mouillage de 24 à 15 p. 100 d'eau ajoutée respectivement. Ce patron mouillait son lait de façon routinière, et continue depuis on ne sait combien de temps. Les billes hydromètres de Golding, la preuve en est faite ici, permettraient de déceler un mouillage grossier du lait au-delà d'un seuil défini, ou à définir.

Un tel décelage permettant d'assortir les laits ayant une densité trop faible pourrait se faire à l'aide d'une seule bille hydromètre ayant une densité de soit 1,026 ou 1,025 à $68^{\circ} \mathrm{F}$. Une telle bille de couleur rouge, c'est-à-dire très visible dans le lait, et d'un diamètre assez grand, soit deux pouces, pourrait être placée dans le bassin de pesée à la réception. Un treillis, empêchant la bille de sortir avec le lait, pourrait se placer au fond du bassin, et en fait la bille pourrait être maintenue en place à une position donnée dans le bassin par des $\mathrm{f} l \mathrm{ls}$ de plastique allouant suffisamment de jeu pour qu'elle puisse flotter ou non dans le lait selon la densité du lait.

Ainsi, de même qu'on note la température du lait à la réception, on pourrait également noter la densité. Tous les laits où la bille a coulé pourraient être soumis à l'épreuve au cryoscope pour fin de confirmation quantitative du mouillage, en employant la méthode que nous suggérions précédemment [4].

On observe aux tableaux dans tous les cas, sans exception, que la teneur en S.N.G. par la méthode Golding est inférieure à la teneur en S.N.G. par gravimétrie. Puisque nous ne mettons pas en doute ici la validité des résultats obtenus par la méthode gravimétrique, nous sommes convaincus que décidément, l'équation qui a servi à préparer les valeurs précalculées utilisées pour la conversion n'étaient pas tout à fait applicables à nos conditions.

Les différences moyennes entre les teneurs en S.N.G. par les deux méthodes sont les suivantes :

$0,2(9 / 9 / 63), 0,5(10 / 9 / 63), 0,5 \quad(11 / 9 / 63), 0,3(12 / 9 / 63)$ et 0,4 $(6 / 11 / 63)$. La différence moyenne pour les cinq séries est la suivante : 0,36 ou 0,4 .

\section{Discussion}

D'une part, Jenness et Patton [8], devant le grand nombre d'équations suggérées pour convertir de densité à teneur en S.N.G., ont conclu à une commune observation relative à toutes ces équations. Cette observation est que les équations ne sont applicables qu'aux donnéos desquelles elles furent dérivées. 


\section{TABLEAU 4}

RÉSULTATS DE DÉTERMINATION DE LA TENEUR EN S.N.G. PAR LA MÉTHODE GOLDING EN COMPARAISON A LA MÉTHODE GRAVIMÉTRIQUE

(12 septembre 1963)

\begin{tabular}{|c|c|c|c|c|c|c|}
\hline \multirow[b]{2}{*}{ Numéro } & \multicolumn{3}{|c|}{$P A R$ DIFFERENCE } & \multicolumn{2}{|c|}{$G O L D I N G$} & \multirow[b]{2}{*}{ Différence } \\
\hline & $\begin{array}{l}\text { Gras \% } \\
\text { Babcock }\end{array}$ & $\begin{array}{c}\text { S.T. \% } \\
\text { (Mojonnier }\end{array}$ & $\begin{array}{l}\text { S.N.G. \% } \\
\text { (Diffé- } \\
\text { ) rence) }\end{array}$ & $\begin{array}{l}\text { No de } \\
\text { billes } \\
\text { au fond }\end{array}$ & $\begin{array}{c}\text { S.N.G. \% } \\
\text { Corres- } \\
\text { pondants }\end{array}$ & \\
\hline 1 & 3,4 & 12,10 & 8,7 & 6 & 8,5 & 0,2 \\
\hline 2 & 3,4 & 12,12 & 8,7 & 7 & 8,2 & 0,5 \\
\hline 3 & 3,0 & 11,48 & 8,5 & 7 & 8,1 & 0,4 \\
\hline 4 & 3,9 & 12,51 & 8,6 & 7 & 8,4 & 0,2 \\
\hline 5 & 3,4 & 12,18 & 8,8 & 6 & 8,5 & 0,3 \\
\hline 6 & 3,6 & 12,55 & 8,9 & 6 & 8,6 & 0,3 \\
\hline 7 & 3,9 & 13,12 & 9,2 & 5 & 8,9 & 0,3 \\
\hline 8 & 3,7 & 12,18 & 8,5 & 7 & 8,3 & 0,2 \\
\hline 9 & 3,9 & 13,13 & 9,2 & 4 & 9,2 & 0,0 \\
\hline 10 & 3,3 & 12,35 & 9,0 & 5 & 8,8 & 0,2 \\
\hline 11 & 3,4 & 12,07 & 8,7 & 7 & 8,2 & 0,5 \\
\hline 12 & 3,6 & 12,33 & 8,7 & 7 & 8,3 & 0,4 \\
\hline
\end{tabular}

Moyenne: $\quad 0,3$

D'autre part, Erb, Manus et Ashworth [3], (pour le total des échantillons analysés) avaient dérivé l'équation présentée plus haut, d'observations sur des échantillons de lait des trois races laitières : Guernesey, Holstein et Jersey, dont une équation particulière avait été dérivée pour chacune d'elles. Dans un travail plus récent, quelques 57 équations différentes sont présentées par Erb [1], selon que dérivées de résultats groupés en classes différentes. Des équations (lesquelles seraient beaucoup plus satisfaisantes) furent dérivées, tenant compte de la teneur en gras, alors que d'autres le furent selon la race laitière, avec variation de la teneur en gras en dedans de limites définies. Erb [1] croit que les équations dérivées d'observations classées par races laitières sont les plus satisfaisantes. Puisque nous ne connaissons pas la race laitière qui a produit le lait venant à la réception dans nos usines, et que souvent le lait provient de troupeaux croisés, il nous faudrait utiliser les équations dérivées, tenant compte de la teneur en gras, des tables de conversion donnant les valeurs précalculées à cet effet.

Nous pouvons donc assurer que si nos résultats pour les S.N.G. par la méthode Golding sont plus bas que par la méthode gravimétrique, c'est que l'équation et les valeurs précalculées servant 
TABLEAU 5

RÉSULTATS DE DÉTERMINATION DE LA TENEUR EN S.N.G. PAR LA MÉTHODE GOLDING EN COMPARAISON A LA MÉTHODE GRAVIMÉTRIQUE (6 novembre 1963)

\begin{tabular}{|c|c|c|c|c|c|c|}
\hline \multirow[b]{2}{*}{ Numéro } & \multicolumn{3}{|c|}{ PAR DIFFERENCE } & \multicolumn{2}{|c|}{$G O L D I N G$} & \multirow[b]{2}{*}{ Différence } \\
\hline & $\begin{array}{l}\text { Gras \% } \\
\text { Babcock }\end{array}$ & $\begin{array}{c}\text { S.T. \% } \\
\text { (Mojonnier) }\end{array}$ & $\begin{array}{l}\text { S.N.G. \% } \\
\text { (Diffé- } \\
\text { rence) }\end{array}$ & $\begin{array}{l}N o \text { de } \\
\text { billes } \\
\text { au fond }\end{array}$ & $\begin{array}{c}\text { S.N.G. \% } \\
\text { Corres- } \\
\text { pondants }\end{array}$ & \\
\hline 1 & 3,4 & 12,18 & 8,8 & 6 & 8,5 & 0,3 \\
\hline 2 & 3,5 & 12,28 & 8,8 & 6 & 8,5 & 0,3 \\
\hline 3 & 3,3 & 11,93 & 8,6 & 6 & 8,5 & 0,1 \\
\hline 4 & 3,2 & 11,74 & 8,5 & 6 & 8,4 & 0,1 \\
\hline 5 & 3,2 & 11,81 & 8,6 & 7 & 8,2 & 0,4 \\
\hline 6 & 3,3 & 11,86 & 8,6 & 7 & 8,2 & 0,4 \\
\hline 7 & 3,0 & 11,93 & 8,9 & 6 & 8,4 & 0,5 \\
\hline 8 & 3,5 & 12,25 & 8,7 & 7 & 8,2 & 0,5 \\
\hline 9 & 3,3 & 12,04 & 8,7 & 7 & 8,2 & 0,5 \\
\hline 10 & 3,8 & 13,01 & 9,2 & 6 & 8,6 & 0,6 \\
\hline 11 & 3,9 & 13,05 & 9,1 & 6 & 8,7 & 0,4 \\
\hline 12 & 3,1 & 12,02 & 8,9 & 5 & 8,7 & 0,2 \\
\hline
\end{tabular}

de moyens de conversion du nombre de billes à la teneur en S.N.G. n'étaient pas entièrement adaptées à nos conditions.

Utilisant l'équation requise, nos résultats auraient été en moyenne de 0,4 p. 100 plus haut.

Erb, Manus et Ashworth [3] ont indiqué pour l'équation qui nous était disponible $(2,0$ à 9 p. 100 Gras $)$, la teneur en S.N.G. par la méthode Golding différait de la teneur en S.N.G. par gravimètre, de plus de 0,2 p. 100 S.N.G. dans 25 p. 100 des échantillons observés, quoique pour tous les échantillons la différence était inférieure à 0,1 p. 100 S.N.G.

Disposant d'une équation satisfaisante, la différence moyenne pour la teneur en gras obtenue par les deux méthodes n'aurait pas été de 0,4 mais de 0,1 , variation de cet ordre due à l'inaptitude qui est le facteur limite pour ces méthodes, de donner une plus grande précision.

\section{CONCLUSION}

La détermination de la teneur en S.N.G. dans le lait avec des billes hydromètres de Golding offre des avantages certains. Puisque le tableau de conversion fourni avec l'équipement n'est pas adapté 
à nos conditions, il faudrait ajouter 0,4 p. 100 S.N.G. aux résultats, ou autrement il faudrait sans doute préparer de nouveaux tableaux, et cette fơis utiliser-des équations dérivés de résultats obtenus pour des échantillons de lait dont les teneurs en gras seraient comprises entre des limites plus restreintes.

\section{SUMMARY}

The determination of the percentage of solid non fat in milk with Golding's hydrometer balls is much advantageous. Since the conversion panel which is sold with the whole instrument is not adjusted as our conditions of work, it would be necessary to add 0,4 p. 100 S.N.F. to the results; if not, one would undoubtedly have to prepare new panels, and then use equations coming from results achieved with samples of milk whose percentage of fat would be included in more narrow limits.

\section{REMERCIEMENTS}

L'auteur désire tout particulièrement reconnaître la collaboration reçue, au cours de ce projet, de M. Rosaire Samson, technicien et contremaître au laboratoire de la Coopérative à Granby.

\section{BIBLIOGRAPHIE}

[1] R. E. Eвв. Influence of breed and milk fat percentage when estimating solids-not-fat in milk of individual cows by the plastic bead method of Golding. Jour. Dairy Science, [46], 12, 1428, 1963.

[2] R. E. ERB, L. J. MANUS. Estimating solids-not-fat in herd milk using the plastie bead method of Golding. Jour. Dairy Science, [46], 12, 1373, 1963.

[3] R. Erb, L. MANus, U. Ashworth. Comparative Accuracy of the plastic bead method of Golding and the lactometer method of Watson for routine determination of S.N.F. in milk. Jour. Dairy Science [43] $5,605,1960$.

[4] Roger N. Grroux. Effets de préservatifs sur l'abaissement du point de congélation du lait. Le Québec laitier, [22], 9, 10, 1963.

[5] N. S. Golding. A solids-not-fat test for milk using density plastic beads as hydromerers. Jour. Dairy Science, [42], 5, 899. Paper M-23, 1959.

[6] N. S. Golding. Plastic hydrometers (lactometers) for measuring the density of skim and whole milk. XVth. Intern. Dairy Congr., 3, 1568, 1959.

[7] N. S. Golding. Test for solids-not-fat. Milk Prod. Jour. [52], 7, 10, 1961.

[8] R. Jenness, S. Patton. Principles of Dairy Chemistry (p. 243). John Wiley and Sons, 1959.

[9] L. S. MANUS, R. E. ERB. - Comparison of relative accuracy between testing semi-monthly composites or single random samples of herd milk for composition. Jour. Dairy Science [46], 12, 1367, 1963.

[10] Udy Analyser Co. Instructions for using plastic bead hydrometers for the determination of solids-not-fat in milk. 1963. 